\title{
Afatinib in locally advanced/metastatic NSCLC harboring common EGFR mutations, after chemotherapy: a Phase IV study
}

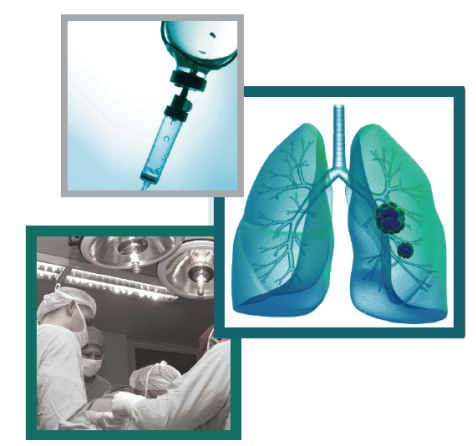

\author{
Sumitra Thongprasert*,1, Sarayut L Geater², Dana Clement ${ }^{3}$, Amr Abdelaziz ${ }^{4}$, Jasmin \\ Reyes-Igama5, Dragana Jovanovic ${ }^{6}$, Aurelia Alexandru ${ }^{7}$, Michael Schenker $^{8}$, Virote \\ Sriuranpong ${ }^{9}$, Piotr Serwatowski ${ }^{10}$, Sheethal Suresh ${ }^{11}$, Agnieszka Cseh $^{12}$ \& Rabab Gaafar $^{13}$ \\ ${ }^{1}$ Medical Oncology Unit, Wattanosoth Hospital \& Bangkok Hospital Chiang Mai (BDMS), Chiang Mai 50000, Thailand \\ ${ }^{2}$ Department of Internal Medicine, Songklanagarind Hospital, Prince of Songkla University, Songkhla 90110, Thailand \\ ${ }^{3}$ Medical Oncology Department, Regional Oncology Institute of lasi, lasi 700483, Romania \\ ${ }^{4}$ Clinical Oncology Department, Alexandria Clinical Research Centre, Alexandria University, Alexandria 21131, Egypt \\ ${ }^{5}$ Department of Internal Medicine, Hematology \& Oncology, Baguio General Hospital \& Medical Center, Baguio City, 2600 \\ Benguet, Philippines \\ ${ }^{6}$ Clinic for Pulmonology, Clinical Center Serbia, Belgrade 11000, Serbia \\ ${ }^{7}$ Medical Oncology II Department, Oncology Institute of Bucharest, Bucharest 022328, Romania \\ ${ }^{8}$ Oncology, Oncology Center of Sf Nectarie Ltd, Craiova, Romania \\ ${ }^{9}$ Department of Medicine, Faculty of Medicine, Chulalongkorn University \& The King Chulalongkorn Memorial Hospital, Bangkok \\ 10330, Thailand \\ ${ }^{10}$ Department of Clinical Oncology \& Chemotherapy, Specialist Hospital, Szczecin-Zdunowo 70-891, Poland \\ ${ }^{11}$ Oncology Department, Boehringer Ingelheim Pharmaceuticals, Inc., Singapore 199555, Republic of Singapore \\ ${ }^{12}$ Medical Department, Boehringer Ingelheim RCV GmbH \& Co. KG, Vienna 1120, Austria \\ ${ }^{13}$ Medical Oncology Department, National Cancer Institute, Cairo University, Cairo 11796, Egypt \\ *Author for correspondence: Tel.: +66 539 45480; Fax: +66 532 89232; Sumitra95@gmail.com
}

\begin{abstract}
Aim: The current study evaluated the efficacy and tolerability of second-line afatinib in patients with EGFR mutation-positive (EGFRm+) non-small-cell lung cancer (NSCLC) following chemotherapy. Patients \& methods: In this open-label, single-arm Phase IV study, patients with EGFRm+ (Del19/L858R) NSCLC who had progressed following platinum-based chemotherapy received afatinib (starting dose $40 \mathrm{mg} /$ day). The primary end point was confirmed objective response. Results: 60 patients received afatinib for a median duration of 11.5 months. $50 \%$ of patients had a confirmed objective response, of median duration 13.8 months. Median progression-free survival was 10.9 months. The most common treatment-related adverse events were diarrhea $(72 \%)$, rash $(28 \%)$ and paronychia $(23 \%)$. Conclusion: Our data support the use of afatinib (40 mg/day) as an effective and well-tolerated second-line treatment in EGFRm+ NSCLC.
\end{abstract}

First draft submitted: 17 April 2019; Accepted for publication: 16 July 2019; Published online:

2 September 2019

Keywords: afatinib • EGFR • second line

The oral, irreversible ErbB family blocker afatinib is approved in the USA and the EU for first-line treatment of $E G F R$ mutation-positive $(E G F R \mathrm{~m}+)$ locally advanced or metastatic non-small-cell lung cancer (NSCLC), the recommended dose being $40 \mathrm{mg} /$ day [1-4]. In the EU, afatinib is also approved as a second-line treatment option for EGFR tyrosine kinase inhibitor (TKI)-naive patients with EGFRm + NSCLC that has progressed on or after platinum-based chemotherapy [2]. Approval of first-line afatinib was based on the results of two large, randomized, Phase III studies (LUX-Lung 3 and LUX-Lung 6) and was further supported by results of the Phase IIb LUX-Lung 7 study. In patients with EGFRm + NSCLC, these studies showed superior efficacy and patient-reported outcomes with first-line afatinib compared with standard platinum-doublet chemotherapy (LUX-Lung 3 and LUX-Lung 6) and the reversible first-generation EGFR TKI gefitinib (LUX-Lung 7) [5-7]. In addition, the LUX-Lung 2 study provided data to support the use of second-line afatinib in patients with EGFRm + NSCLC progressing following first-line chemotherapy [8]. LUX-Lung 2 included patients with lung adenocarcinoma who had received no more than one round of chemotherapy and had mutations in exons 18-21 of the EGFR gene. Afatinib was

Future Medicine 
administered as second-line therapy to 68 patients, 61 of whom received a dose of $50 \mathrm{mg} /$ day and seven of whom received $40 \mathrm{mg} /$ day [8]. A total of 39 of $68(57 \%)$ patients had a confirmed objective response (OR) [8]. Median progression-free survival (PFS) by independent review was 8.0 months and median overall survival was 23.3 months [8].

While the benefits of first-line afatinib have been shown, chemotherapy is still commonly used as first-line therapy. Consequently, use of afatinib as second-line therapy remains common in patients with EGFRm+ NSCLC. The current single-arm, open-label Phase IV trial was designed to assess the efficacy and safety of afatinib $40 \mathrm{mg} / \mathrm{day}$ as second-line therapy in EGFR TKI-naive patients with advanced/metastatic NSCLC and common EGFR mutations (Del19 and/or L8585R) who had progressed following first-line platinum-based chemotherapy.

\section{Materials \& methods}

\section{Study design \& patients}

This multicenter, open-label, single-arm Phase IV study was performed across 24 sites in seven countries (Egypt, Malaysia, Philippines, Poland, Romania, Serbia and Thailand). Eligible patients were aged 18 years or older, and had an Eastern Cooperative Oncology Group performance status of 0 or 1, a pathologically confirmed diagnosis of stage IIIB or IV adenocarcinoma of the lung (American Joint Committee on Cancer version 6), measurable disease according to Response Evaluation Criteria in Solid Tumors (RECIST, version 1.1) [9], radiologically confirmed progression or recurrence of disease during or following first-line therapy with a platinum-based chemotherapy regimen, and documented presence of a common EGFR mutation (L858R and/or Del19), with no other known $E G F R$ mutations. Patients had to have recovered from any previous therapy-related toxicity to grade 1 or less, or grade 2 or less for alopecia and stable sensory neuropathy, as defined by the National Cancer Institute Common Terminology Criteria for Adverse Events (NCI CTCAE, version 3.0) [10], and to have a life expectancy of at least 3 months. Patients were excluded if they had previously received: more than one line of prior therapy, not counting radiotherapy, radiosensitizers and/or intrapleural administration of anticancer agents; less than three cycles of platinum-based chemotherapy due to toxicity and/or intolerance of treatment; any EGFR-targeting TKI or antibody; chemotherapy, biological therapy or investigational agents within 3 weeks of the start of trial treatment; hormonal therapy within 2 weeks; radiotherapy within 4 weeks (except for palliative radiation to target organs other than the chest, which was allowed up to 2 weeks prior to the trial, and single-dose palliative treatment for symptomatic metastasis [to be discussed with the sponsor before enrollment in the study]); or major surgery within 4 weeks of starting trial treatment, or surgery scheduled during the projected course of the trial.

The study protocol was designed in accordance with the principles of the Declaration of Helsinki, the International Conference on Harmonization Guideline for Good Clinical Practice, and applicable regional requirements. All patients provided written informed consent to participate in the trial.

\section{Procedures}

Eligible patients received afatinib $40 \mathrm{mg}$ orally once daily. If patients had any drug-related adverse events (AEs) grade $\geq 3$, or grade 2 diarrhea for more than 2 days, and/or a rash for more than 7 days, then the study treatment was interrupted until recovery to grade 1 or less, following which, the dose was reduced. Reductions in dose to $30 \mathrm{mg} /$ day and then to $20 \mathrm{mg}$ /day were allowed; if a patient could not tolerate $20 \mathrm{mg} /$ day, afatinib was permanently discontinued. Dose increases were not permitted. Patients were treated with afatinib until disease progression or discontinuation for other reasons. However, patients who had obtained clinical benefit (as judged by the investigator and based on a careful clinical assessment) could continue on afatinib beyond radiological progression.

Assessment of EGFR mutations was based on an existing analysis, conducted locally, or if this was not available, was performed locally on a tumor biopsy using a validated technique, or sent to the central laboratory for analysis. Tumors were assessed by CT scan or MRI every 8 weeks until week 56, and then every 12 weeks until documented progression or discontinuation of treatment. Safety laboratory assessments (urine analysis, biochemistry and hematology) were done locally at screening, on the first visit of each treatment cycle, and at the end of treatment.

\section{Outcomes}

The primary end point was confirmed objective tumor response (OR), defined as complete response (CR) or partial response (PR) (response outcomes were assessed by investigators according to RECIST version 1.1) [9]. Secondary end points were PFS, defined as the time from treatment start to disease progression or death, and disease control, 
Table 1. Patient disposition.

\begin{tabular}{|c|c|c|}
\hline \multirow[t]{2}{*}{ Characteristic } & \multicolumn{2}{|c|}{ Afatinib $40 \mathrm{mg} /$ day } \\
\hline & $\mathbf{n}$ & $(\%)$ \\
\hline Enrolled & 70 & \\
\hline Not entered & 10 & \\
\hline Entered & 60 & $(100.0)$ \\
\hline Not treated & 0 & \\
\hline Treated & 60 & $(100.0)$ \\
\hline Patients discontinued from afatinib ${ }^{\dagger}$ & 60 & $(100.0)$ \\
\hline Reasons for discontinuation: & & - \\
\hline - Progressive disease according to RECIST & 24 & $(40.0)$ \\
\hline - Clinical signs and symptoms of progression & 2 & $(3.3)$ \\
\hline Adverse events $\ddagger$ & 12 & $(20.0)$ \\
\hline Noncompliant with protocol & 1 & $(1.7)$ \\
\hline Lost to follow-up & 0 & $(0.0)$ \\
\hline Refused to continue afatinib & 1 & $(1.7)$ \\
\hline Other ${ }^{\dagger}$ & 20 & (33.3) \\
\hline \multicolumn{3}{|c|}{$\begin{array}{l}\text { † Due to trial completion, all patients are reported as having discontinued afatinib. However, } 20 \text { patients, marked as 'other', were still continuing to derive } \\
\text { benefit from second-line afatinib and therefore continued to receive afatinib outside of the clinical trial, in accordance with the drug label. } \\
\text { †Four other patients discontinued treatment due to AEs, but were reclassified as having symptoms of disease progression, rather than AEs, and so are } \\
\text { not listed here. } \\
\text { AE: Adverse event; RECIST: Response Evaluation Criteria In Solid Tumors. }\end{array}$} \\
\hline
\end{tabular}

defined as CR, PR or stable disease (SD) [9]. The safety and tolerability of afatinib was assessed on the basis of the incidence and intensity of AEs, graded according to CTCAE version 3.0 [10].

\section{Statistical analysis}

Efficacy and safety were evaluated in a descriptive manner and no formal statistical hypothesis tests were used. The treated dataset (encompassing all patients who were documented to have taken at least one dose of afatinib) was used for efficacy and safety analyses, calculation of demographic data, and assessment of exposure. The proportions of patients with an OR and with disease control were calculated; 95\% CIs were calculated using the ClopperPearson formula. For patients with an OR, the time to response and duration of response were determined using Kaplan-Meier analysis; for patients with disease control, the duration of disease control was also calculated using Kaplan-Meier analysis. For assessment of PFS, patients without disease progression who were still on afatinib at database lock were censored. Median PFS was calculated using Kaplan-Meier analysis. For safety analyses, exposure was assessed according to the duration of treatment. All AE analyses were descriptive and based on the number of patients with AEs.

In the Phase II LUX-Lung 2 study [8], a confirmed investigator-assessed PR rate of 60\% was reported among the 68 patients receiving second-line afatinib. In the current study, with 60 patients and a predicted response rate of $60 \%$, the $95 \%$ CI of the response rate was anticipated to be $47-72 \%$ based on the Clopper-Pearson exact method. Thus, a sample size of 60 patients was considered to provide reasonable precision for estimation of the response rate.

This study is registered with ClinicalTrials.gov (NCT02208843).

\section{Results}

Between 9 October 2014 and 13 June 2017, 70 patients were enrolled across 24 sites (Egypt, three sites; Malaysia, one site; Philippines, three sites; Poland, four sites; Romania, five sites; Serbia, four sites; Thailand, four sites). Of these, 60 patients received at least one dose of afatinib $40 \mathrm{mg} /$ day (Table 1). Baseline characteristics for these patients are shown in Table 2. Briefly, 27 (45\%) patients were male, most were white (41 patients [68\%]), and the mean age was 59.9 years. All patients had $E G F R \mathrm{~m}+$ NSCLC, having either an L858R mutation (20 patients [33\%]) or a Del19 mutation only (38 patients [63\%]), or both mutations simultaneously (two patients [3\%]). Almost all patients (57 patients [95\%]) had stage IV NSCLC at screening, and all patients had received prior chemotherapy. The mean time from first diagnosis was 12.8 months (range 2-192 months; median 8.4 months). Nine patients 
Table 2. Baseline demographic and clinical characteristics.

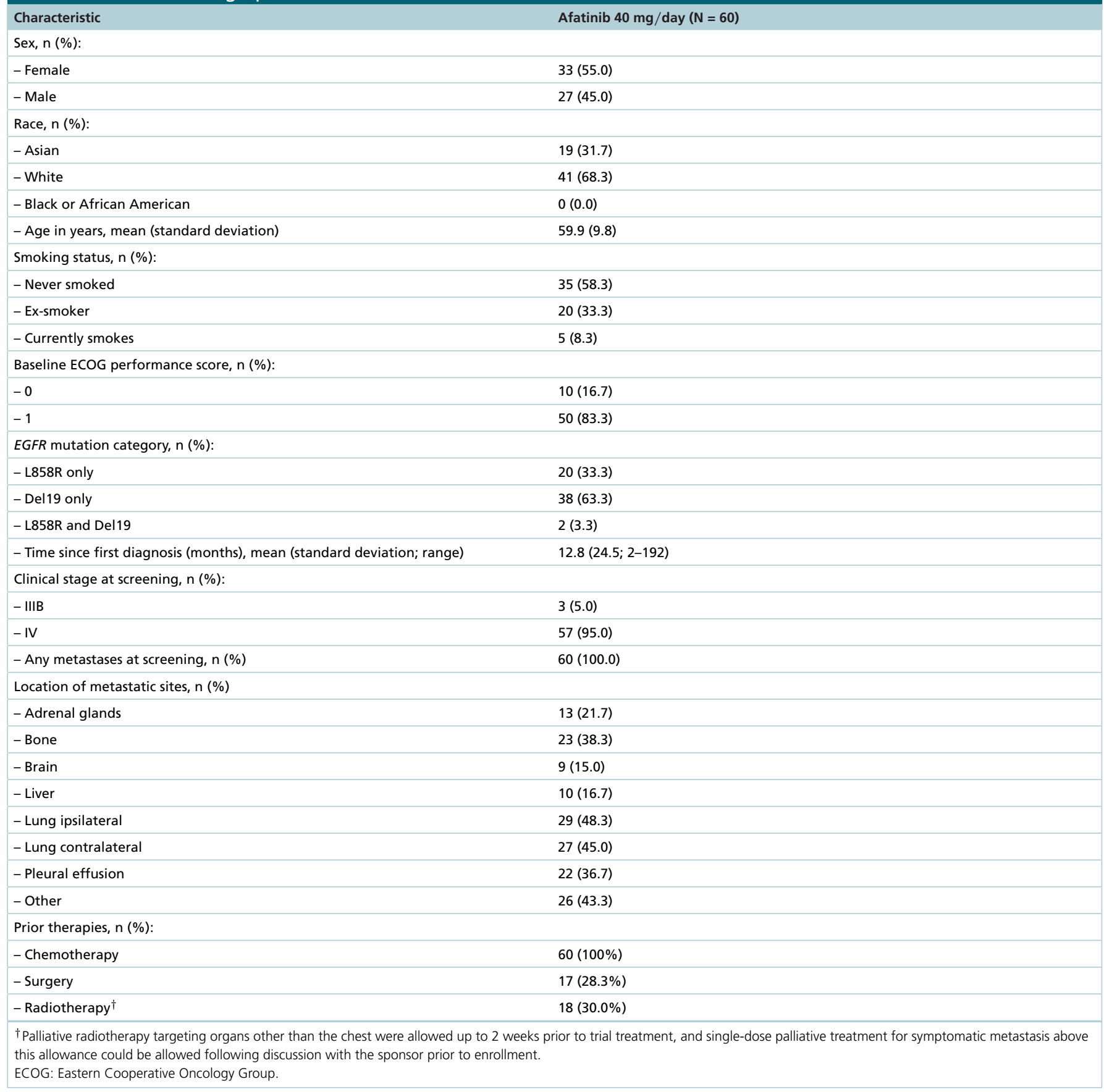

ECOG: Eastern Cooperative Oncology Group.

(15\%) had brain metastases at screening, seven of whom had received whole brain irradiation (WBRT), one patient had undergone stereotactic radiation, and one had no brain radiotherapy.

All patients received afatinib at a starting dose of $40 \mathrm{mg} /$ day. Overall, 52 (87\%) patients were treated for at least 90 days, and the median duration of treatment was 11.5 months. On completion of the study, 38 patients had discontinued afatinib due to disease progression or AEs. One patient had discontinued treatment by choice and one patient had been discontinued due to noncompliance with the study protocol. Twenty patients were continuing to derive benefit from second-line afatinib, and therefore continued treatment with afatinib outside of the trial, in accordance with the drug label (Table 1). Two patients with baseline brain metastases (one of whom had previously 
Table 3. Best confirmed treatment response.

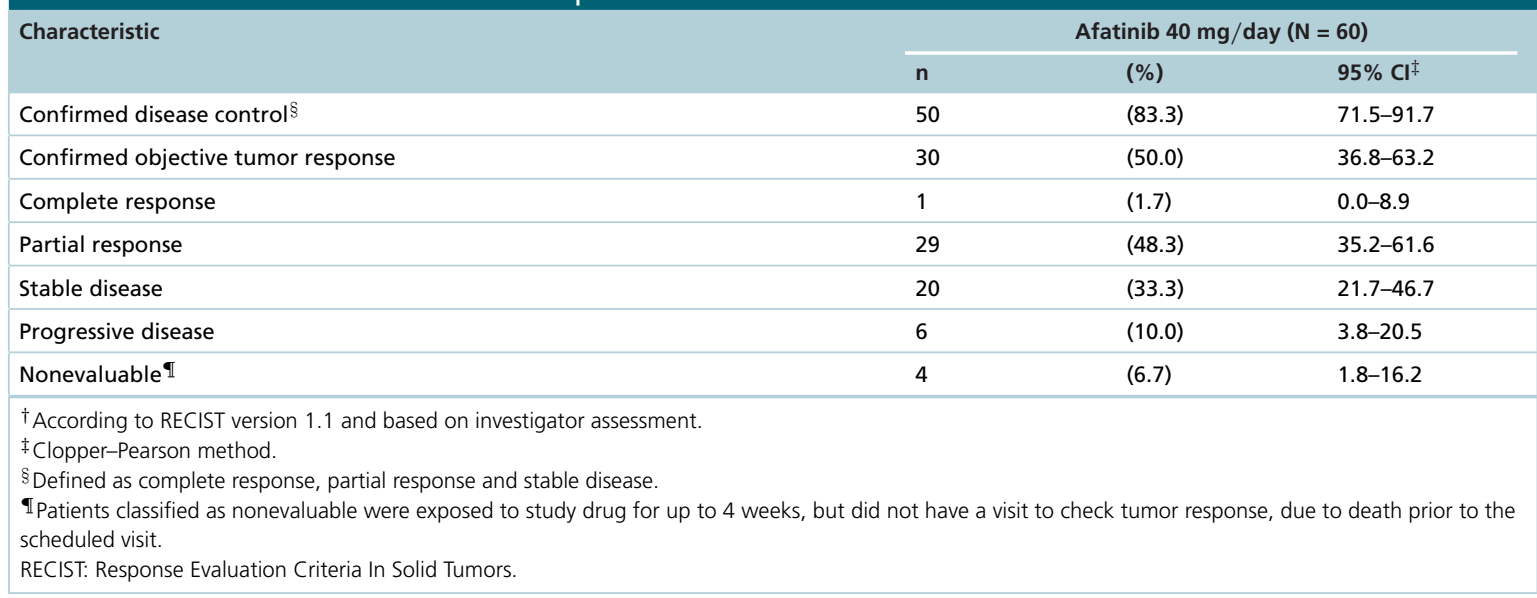

\section{Table 4. Safety and tolerability findings.}

Number of patients, $\mathrm{n}(\%)$

\begin{tabular}{|l|l|}
\hline Patients with any AE & $57(95.0)$ \\
\hline Patients with afatinib-related ${ }^{\dagger}$ AEs & $55(91.7)$ \\
\hline Patients with AEs leading to afatinib dose reduction & $25(41.7)$ \\
\hline Patients with AEs leading to afatinib discontinuation ${ }^{\ddagger}$ & $12(20.0)$ \\
\hline Patients with other significant AEs (according to ICH E3 ${ }^{\S}$ ) & $25(41.7)$ \\
\hline Patients with serious AEs $\mathbb{I}$ & $21(35.0)$ \\
\hline Patients with AEs by highest CTCAE grade & $32(53.3)$ \\
\hline - Grades 1 or 2 & $25(41.7)$ \\
\hline - Grades $\geq 3$ & 3
\end{tabular}

${ }^{\dagger}$ As defined by the investigator.

‡Four additional patients discontinued treatment due to AEs, but were reclassified as having symptoms of disease progression, rather than AEs, so are not listed here.

§Guideline from the International Conference on Harmonization of Technical Requirements for Registration of Pharmaceuticals for Human Use (ICH) on the structure and content of clinical study reports.

I Patients could be counted in more than one category of serious AE.

AE: Adverse event; CTCAE: Common Terminology Criteria for Adverse Events.

received WBRT) remained on treatment for 12 months (without evidence of disease progression in the brain) and then switched to commercially available afatinib.

The primary study end point of confirmed OR (as assessed by the investigators) was achieved by 30 patients (50\%), with one patient (2\%) achieving a confirmed CR and 29 (48\%) a confirmed PR (Table 3). The median duration of response was 13.8 months (95\% CI: 9.16-18.88; Figure 1A). Six of nine patients with brain metastases at baseline $(67 \%)$ achieved a confirmed PR, two $(22 \%)$ had stable disease, and one (11\%) had progressive disease. Only one patient developed a new brain lesion following 8 weeks of afatinib exposure, although this was asymptomatic and did not lead to treatment discontinuation. This patient had a history of brain metastases and had previously received WBRT; they continued to receive treatment following disease progression until death $(\sim 5$ months from the start of treatment). Among the 51 patients with no history of brain metastases, four patients developed new brain lesions, 131, 240, 299 and 632 days following the start of treatment.

Regarding the secondary efficacy end points of PFS and disease control, 39 patients (65\%) had an event contributing to the PFS analysis (i.e., disease progression as determined by investigator assessment or death); median PFS was 10.9 months (95\% CI: 6.4-13.2; Figure 1B). Fifty patients (83\%) showed confirmed disease control, with a median duration of disease control of 11.9 months (95\% CI: 10.8-20.7; Figure 1C).

Ninety-five percent of patients had at least one AE during the study (Table 4). A total of 32 patients (53\%) had AEs of grade 1 or 2 , while 25 (42\%) experienced AEs grade $\geq 3$. More than $90 \%$ of patients $(92 \%)$ had at least one AE deemed by the investigator to be related to treatment (Table 4). The most commonly occurring drug-related 
(A)

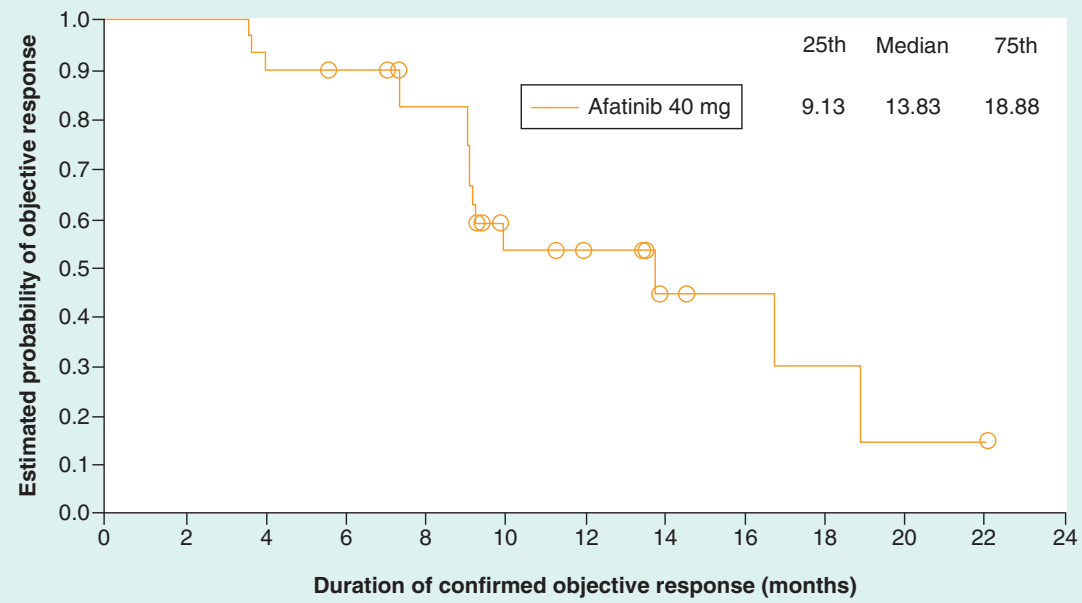

Number at risk

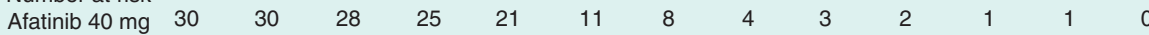

(B)

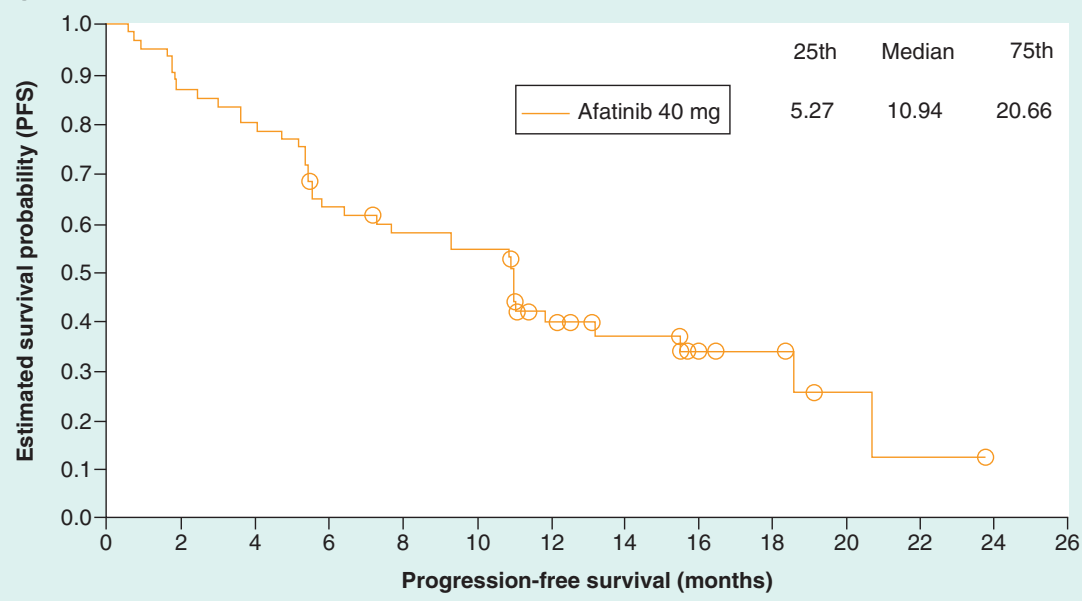

Number at risk

$\begin{array}{lllllllllllllll}\text { Afatinib } 40 \mathrm{mg} & 60 & 52 & 48 & 37 & 33 & 31 & 17 & 13 & 7 & 6 & 2 & 1 & 0 & 0\end{array}$

(c)

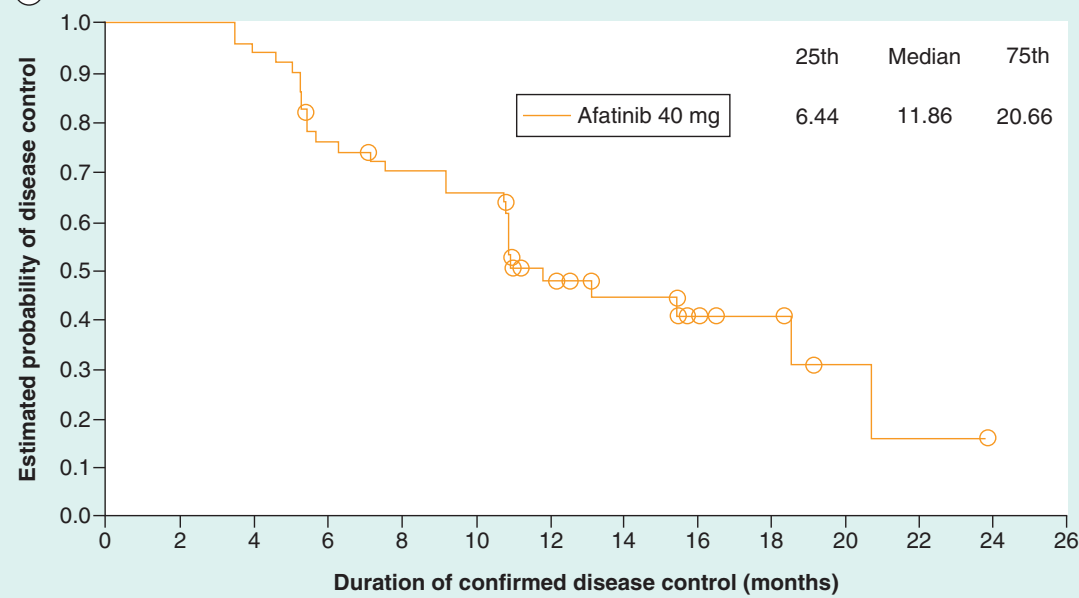

Number at risk

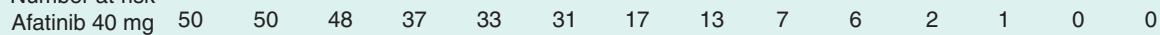

Figure 1. Kaplan-Meier analysis of key study end points. Kaplan-Meier curves of (A) duration of objective response, (B) PFS, and (C) disease control, all based on investigator assessment.

PFS: Progression-free survival. 
Table 5. Afatinib-related AEs with an incidence of at least $10 \%$.

\begin{tabular}{|c|c|c|c|}
\hline MedDRA preferred term, $\mathrm{n}(\%)$ & All Grades ${ }^{\dagger}$ & Grade 3 & Grade 4 \\
\hline Overall drug-related AEs & $55(91.7)$ & $12(20.0)$ & $3(5.0)$ \\
\hline Diarrhea & $43(71.7)$ & $6(10.0)$ & 0 \\
\hline Rash/acne $e^{\ddagger}$ & $35(58.3)$ & $2(3.4)$ & 0 \\
\hline Rash & $17(28.3)$ & $1(1.7)$ & 0 \\
\hline Dermatitis acneiform & $10(16.7)$ & 0 & 0 \\
\hline Dermatitis & $6(10.0)$ & $1(1.7)$ & 0 \\
\hline Mucosal inflammation & $11(18.3)$ & $4(6.7)$ & 0 \\
\hline Fatigue & $9(15.0)$ & $1(1.7)$ & 0 \\
\hline Anemia & $8(13.3)$ & $1(1.7)$ & 0 \\
\hline Hypokalemia & $8(13.3)$ & $2(3.3)$ & $1(1.7)$ \\
\hline
\end{tabular}

$\dagger$ AEs with grades $1,2,3,4,5$; there were no grade 5 AEs in the study.

$¥$ Grouped terms; these include additional AEs that are not listed due to $<10 \%$ incidence of the preferred terms.

AE: Adverse event; MedDRA: Medical dictionary for regulatory activity.

AEs (any grade) were diarrhea (72\%), rash (28\%), paronychia (23\%), mucosal inflammation (18\%) and dermatitis acneiform (17\%; Table 5). Serious AEs occurred in 21 (35\%) of patients (Table 4); most of these were considered to be serious because the patient had to be hospitalized (14 patients [23\%]). Seven patients (12\%) had serious AEs that were considered to be drug-related by the investigator, with diarrhea being the most common of these (four patients [7\%]).

In total, 25 patients (42\%) required a reduction in the dose of afatinib to $30 \mathrm{mg} /$ day (Table 4); six patients (10\%) required a further reduction to $20 \mathrm{mg} /$ day. The median duration of treatment was 169 days for patients taking afatinib $40 \mathrm{mg} /$ day, 212 days for $30 \mathrm{mg} /$ day, and 135 days for $20 \mathrm{mg}$ /day. Twenty per cent of patients had AEs that led to permanent discontinuation of afatinib (Table 4). The most commonly occurring AEs leading to afatinib dose reduction were diarrhea (six patients [10\%]), rash (four patients [7\%]), and dermatitis and mucosal inflammation (three patients [5\%] each).

Eleven patients (18\%) died during the treatment period, but no deaths were attributed to afatinib treatment. Nine of the 11 patients died due to disease progression, while the other two patients had pre-existing risk factors and died from a cerebrovascular accident and a myocardial infarction, respectively.

\section{Discussion}

The current findings confirm the efficacy of $40 \mathrm{mg} /$ day afatinib as second-line therapy for TKI-naive patients with $E G F R \mathrm{~m}+$ NSCLC, after failure of first-line chemotherapy. Half of the patients had a confirmed OR, and the median duration of response exceeded 1 year. In addition, more than $80 \%$ of patients had confirmed disease control, with a median duration of 12 months. Median PFS was 11 months.

These results are consistent with those of a previous trial of second-line afatinib (the Phase II LUX-Lung 2 trial) in which the OR rate with afatinib (starting dose $40 / 50 \mathrm{mg} /$ day) was $57 \%$, median PFS was 8 months, and median overall survival was 23 months [8]. The benefits of afatinib that we observed are also similar to previously reported outcomes with first-line afatinib. The LUX-Lung 3, LUX-Lung 6 and LUX-Lung 7 trials demonstrated favorable outcomes with regard to PFS (11-14 months), time-to-treatment failure (14 months, LUX-Lung 7 only), OR rate (56-70\%) and disease control (90 and 93\%, LUX-Lung 3 and LUX-Lung 6) [5-7,11].

Nine of the patients enrolled in this study (15\%) had brain metastases at baseline, and good control of the brain metastases was achieved in eight of them. Only one patient had developed a new metastatic brain lesion by the time that systemic progression was detected; the brain lesion was asymptomatic and did not lead to discontinuation of treatment. In addition, most patients with baseline brain metastases achieved disease control; six (67\%) had a confirmed PR, two (22\%) had stable disease, and one (11\%) had disease progression. Five of these patients (55\%) remained on treatment for more than 12 months. While the data available do not allow a comparison of central nervous system (CNS) versus non-CNS progression in patients with baseline brain metastases, our results are similar 
to those from LUX-Lung 3, 6 and 7, all of which allowed enrollment of patients with baseline brain metastases; these trials also demonstrated improvements with afatinib in patients with brain metastases [7,12,13]. In addition, competing risk analyses for patients with baseline brain metastases in LUX-Lung 3 and 6 showed that, during treatment with afatinib, the risk of CNS progression was lower than the risk of non-CNS progression [14]. Overall, results from multiple studies consistently show beneficial effects of afatinib in patients with brain metastases.

No new safety signals were identified; the safety profile of afatinib was similar to that identified in previous large trials of EGFR TKIs [5-8,15-24]. Despite the high incidence of AEs, there were relatively few discontinuations due to AEs; this suggests that clinicians successfully managed these events, with dose reductions due to AEs undertaken in approximately $40 \%$ of patients. Dose reductions did not compromise the total duration of exposure to afatinib.

Potential barriers to the use of EGFR TKIs are present in many countries, which may result in the second-line rather than first-line use of these agents. Barriers include the cost of mutation testing, the availability of samples for testing, and the time required to obtain the results and mutation analysis (usually at least 2 weeks) [25,26]. Further, in some low- and middle-income countries, patients may not be able to easily access molecular testing and first-line targeted therapies due to restrictions in government funding and/or policies. For example, in Egypt in 2018, only out-of-pocket payers and those with national and international private insurance coverage (approximately $10 \%$ of patients) were able to access first-line EGFR TKIs and other biology-driven therapies such as bevacizumab and immune checkpoint inhibitors [27]. A further $35-40 \%$ of patients were able to access EGFR TKIs, but only as second-line therapy. In Malaysia, Thailand and The Philippines, first-line EGFR TKI treatment is generally not reimbursed by the government, making this treatment unattainable for all but wealthier patients. Patients who cannot afford to pay for EGFR TKIs receive first-line chemotherapy, and as a result, do not undergo routine EGFR testing at diagnosis. Testing is generally recommended when the patient progresses on first-line chemotherapy; this testing is either paid for by the patient or sponsored by a pharmaceutical company, and is generally only conducted in the largest cities. For patients with confirmed EGFRm + disease, subsequent EGFR TKI treatment may be paid for by patients, the Charity Sweepstakes Office (The Philippines), or in the case of individuals employed in the government sector, the government.

Even in wealthier countries where EGFR TKIs are approved and reimbursed for the first-line treatment of patients with EGFRm + NSCLC, some physicians continue to administer first-line chemotherapy [28]. This may be initiated without waiting for the results of mutation testing, such as when a patient presents in poor health, in order to prevent further deterioration, or when the preference of the physician/patient at diagnosis is to initiate treatment immediately, in order to halt disease progression. Our results suggest that, in patients with EGFRm+ NSCLC who have progressed on or following chemotherapy, treatment with afatinib can provide significant improvements in outcomes. This supports the use of afatinib as a second-line treatment option after progression on/failure of chemotherapy. Our findings are also of relevance to the smaller group of patients who start chemotherapy and subsequently switch to afatinib treatment, and suggest that prior treatment does not impact on outcomes with afatinib. Together with previous studies of first-/second-line afatinib, these findings highlight the benefits of this treatment option in patients with EGFRm + NSCLC, regardless of prior chemotherapy treatment.

\section{Conclusion}

In conclusion, our findings provide further support for the use of afatinib $40 \mathrm{mg} /$ day as second-line therapy in EGFR TKI-naive patients with EGFRm+ (Del19/L858R) NSCLC. These results confirm those of LUX-Lung 2, and suggest that the recommended dose of afatinib ( $40 \mathrm{mg} /$ day) provides effective treatment for patients with $E G F R \mathrm{~m}+$ NSCLC in the first- or second-line setting, and irrespective of prior chemotherapy exposure.

\footnotetext{
Author contributions

S Thongprasert and A Cseh contributed toward study conception and design. All authors contributed toward data collection, analysis and interpretation. All authors contributed toward writing and revision of the manuscript, are fully responsible for all content and editorial decisions, and have approved the final version.
}

Financial \& competing interests disclosure

SL Geater reports grants and personal fees from AstraZeneca, Roche and Boehringer Ingelheim, and grants from Novartis. D Jovanovic reports personal fees related to a regional advisory board from Boehringer Ingelheim. A Alexandru reports personal fees from Bristol Myers Squibb, Novartis and Sanofi. M Schenker reports grants and personal fees from Boehringer Ingelheim, Astra Zeneca, Roche, Astellas, Bristol Myers Squibb, Novartis, Eli Lilly, Pfizer, AbbVie, Merck, Merck Serono, Bioven, Pharma Mar, 
Regeneron, Eisai, and Amgern, and grants from Mylan. V Sriuranpong reports speaker honoraria from Boehringer Ingelheim. S Suresh and A Cseh report employment from Boehringer Ingelheim. S Thongprasert, D Clement, A Abdelaziz, J Reyes-Igama, P Serwatowski and R Gaafar have nothing to disclose. The authors have no other relevant affiliations or financial involvement with any organization or entity with a financial interest in or financial conflict with the subject matter or materials discussed in the manuscript apart from those disclosed.

Medical writing assistance, supported financially by Boehringer Ingelheim, was provided by H Dbouk, of GeoMed, an Ashfield company, part of UDG Healthcare, plc., during the preparation of this article.

\section{Data sharing statement}

The authors certify that this manuscript reports original clinical trial data. Clinical trial registration number: NCT 02208843. The datasets generated and analyzed during the study are available from Sumitra Thongprasert on reasonable request.

\section{Open access}

This work is licensed under the Attribution-NonCommercial-NoDerivatives 4.0 Unported License. To view a copy of this license, visit http://creativecommons.org/licenses/by-nc-nd/4.0/

\section{Ethical conduct of research}

The study protocol was designed in accordance with the principles of the Declaration of Helsinki, the International Conference on Harmonization Guideline for Good Clinical Practice and applicable regional requirements. All patients provided written informed consent to participate in the trial. Clinicaltrials.gov NCT02208843.

\section{Summary points}

- The $40 \mathrm{mg} /$ day afatinib dose was assessed in EGFR mutation-positive non-small-cell lung cancer patients progressing on chemotherapy.

- A total of 60 patients received at least one dose of afatinib $40 \mathrm{mg} /$ day.

- Most patients $(87 \%)$ were treated with afatinib for at least 90 days, and median duration of treatment was 11.5 months.

- Objective response was achieved by $50 \%$ of patients; median duration of response was 13.8 months.

- A total of $83 \%$ of patients had disease control, with median duration 11.9 months.

- Good control of brain metastases was achieved by eight/nine patients with baseline brain metastases.

- Median progression-free survival was 10.9 months.

- Most common drug-related adverse events were diarrhea, rash and paronychia.

- Our data support the use of second-line afatinib in patients with EGFR mutation-positive non-small-cell lung cancer.

\section{References}

Papers of special note have been highlighted as: $\bullet$ of interest; $\bullet \bullet$ of considerable interest

1. Gilotrif ${ }^{\circledR} /{ }^{T M}$ prescribing information. Boehringer Ingelheim (2013). www.accessdata.fda.gov/drugsatfda_docs/label/2013/201292s000lbl.pdf

2. Boehringer Ingelheim. Giotrif $20 \mathrm{mg} / 30 \mathrm{mg} / 40 \mathrm{mg} / 50 \mathrm{mg}$ filmcoated tablets, summary of product characteristics (2016). www.medicines.org.uk/emc/medicine/28353

3. Giordano P, Manzo A, Montanino A et al. Afatinib: an overview of its clinical development in non-small-cell lung cancer and other tumors. Crit. Rev. Oncol. Hematol. 97, 143-151 (2016).

4. Solca F, Dahl G, Zoephel A et al. Target binding properties and cellular activity of afatinib (BIBW 2992), an irreversible ErbB family blocker. J. Pharmacol. Exp. Ther. 343(2), 342-350 (2012).

5. Sequist LV, Yang JC, Yamamoto N et al. Phase III study of afatinib or cisplatin plus pemetrexed in patients with metastatic lung adenocarcinoma with EGFR mutations. J. Clin. Oncol. 31(27), 3327-3334 (2013).

- Primary results from the LUX-Lung 3 Phase III study demonstrating prolonged progression-free survival with first-line afatinib versus cisplatin plus pemetrexed in patients with $E G F R$ mutation-positive non-small-cell lung cancer.

6. Wu YL, Zhou C, Hu CP et al. Afatinib versus cisplatin plus gemcitabine for first-line treatment of Asian patients with advanced non-small-cell lung cancer harbouring EGFR mutations (LUX-Lung 6): an open-label, randomised Phase III trial. Lancet Oncol. 15(2), 213-222 (2014).

7. Park K, Tan EH, O’Byrne K et al. Afatinib versus gefitinib as first-line treatment of patients with EGFR mutation-positive non-small-cell lung cancer (LUX-Lung 7): a Phase IIB, open-label, randomised controlled trial. Lancet Oncol. 17(5), 577-589 (2016). 
8. Yang JC-H, Shih J-Y, Su W-C et al. Afatinib for patients with lung adenocarcinoma and epidermal growth factor receptor mutations (LUX-Lung 2): a Phase II trial. Lancet Oncol. 13(5), 539-548 (2012).

-. Primary results from the LUX-Lung 2 trial which demonstrated efficacy of second-line afatinib in patients with lung adenocarcinoma harboring EGFR mutations in exons 18-21.

9. Eisenhauer EA, Therasse P, Bogaerts J et al. New response evaluation criteria in solid tumours: revised RECIST guideline (version 1.1). Eur. J. Cancer 45(2), 228-247 (2009).

10. National Cancer Institute. Common terminology criteria for adverse events v3.0 (CTCAE) (2009). https://ctep.cancer.gov/protocoldevelopment/electronic_applications/docs/ctcaev3.pdf

11. Paz-Ares L, Tan EH, O'Byrne $\mathrm{K}$ et al. Afatinib versus gefitinib in patients with EGFR mutation-positive advanced non-small-cell lung cancer: overall survival data from the Phase IIb LUX-Lung 7 trial. Ann. Oncol. 28(2), 270-277 (2017).

12. Schuler M, Wu YL, Hirsh V et al. First-line afatinib versus chemotherapy in patients with non-small cell lung cancer and common epidermal growth factor receptor gene mutations and brain metastases. J. Thorac. Oncol. 11(3), 380-390 (2016).

- Post hoc analysis showing clinical activity among patients with baseline brain metastases who received first-line afatinib in the LUX-Lung 3 and LUX-Lung 6 trials.

13. Hochmair M. Medical treatment options for patients with epidermal growth factor receptor mutation-positive non-small cell lung cancer suffering from brain metastases and/or leptomeningeal disease. Target. Oncol. 13(3), 269-285 (2018).

14. Girard N. Optimizing outcomes in EGFR mutation-positive NSCLC: which tyrosine kinase inhibitor and when? Future Oncol. 14(11), 1117-1132 (2018).

15. Mitsudomi T, Morita S, Yatabe Y et al. Gefitinib versus cisplatin plus docetaxel in patients with non-small-cell lung cancer harbouring mutations of the epidermal growth factor receptor (WJTOG3405): an open label, randomised Phase III trial. Lancet Oncol. 11(2), 121-128 (2010).

16. Mok TS, Wu YL, Thongprasert S et al. Gefitinib or carboplatin-paclitaxel in pulmonary adenocarcinoma. N. Engl. J. Med. 361(10), 947-957 (2009).

17. Rosell R, Carcereny E, Gervais R et al. Erlotinib versus standard chemotherapy as first-line treatment for European patients with advanced EGFR mutation-positive non-small-cell lung cancer (EURTAC): a multicentre, open-label, randomised Phase III trial. Lancet Oncol. 13(3), 239-246 (2012).

18. Soria JC, Ohe Y, Vansteenkiste J et al. Osimertinib in untreated EGFR-mutated advanced non-small-cell lung cancer. $N$. Engl. J. Med. 378(2), 113-125 (2018).

19. Sullivan I, Planchard D. Next-generation EGFR tyrosine kinase inhibitors for treating EGFR-mutant lung cancer beyond first line. Front. Med. (Lausanne) 3, 76 (2016).

20. Wu Y-L, Cheng Y, Zhou X et al. Dacomitinib versus gefitinib as first-line treatment for patients with EGFR-mutation-positive non-small-cell lung cancer (ARCHER 1050): a randomised, open-label, Phase III trial. Lancet Oncol. 18(11), 1454-1466 (2017).

21. Zhou C, Wu YL, Chen G et al. Erlotinib versus chemotherapy as first-line treatment for patients with advanced EGFR mutation-positive non-small-cell lung cancer (OPTIMAL, CTONG-0802): a multicentre, open-label, randomised, Phase III study. Lancet Oncol. 12(8), 735-742 (2011).

22. Miller VA, Hirsh V, Cadranel $\mathrm{J}$ et al. Afatinib versus placebo for patients with advanced, metastatic non-small-cell lung cancer after failure of erlotinib, gefitinib, or both, and one or two lines of chemotherapy (LUX-Lung 1): a Phase IIb/III randomised trial. Lancet Oncol. 13(5), 528-538 (2012).

23. Schuler M, Fischer JR, Grohe $\mathrm{C}$ et al. Experience with afatinib in patients with non-small cell lung cancer progressing after clinical benefit from gefitinib and erlotinib. Oncologist 19(10), 1100-1109 (2014).

24. Soria J-C, Felip E, Cobo M et al. Afatinib versus erlotinib as second-line treatment of patients with advanced squamous cell carcinoma of the lung (LUX-Lung 8): an open-label randomised controlled Phase III trial. Lancet Oncol. 16(8), 897-907 (2015).

25. Korpanty G, Leighl NB. Challenges in NSCLC molecular testing (2012). www.oncologyex.com/pdf/vol11_no4/comment_nsclc-molecular-testing.pdf

- Article describing barriers to implementation of molecular testing in non-small-cell lung cancer, which may contribute to the continued use of chemotherapy as first-line treatment.

26. Ellis PM, Verma S, Sehdev S, Younus J, Leighl NB. Challenges to implementation of an epidermal growth factor receptor testing strategy for non-small-cell lung cancer in a publicly funded healthcare system. J. Thorac. Oncol. 8(9), 1136-1141 (2013).

27. C.A.I.R.O. Journal Club Executive Board. The state of lung cancer in Egypt: progress is at hand (2018).www.lungcancernews.org/2018/12/13/the-state-of-lung-cancer-in-egypt-progress-is-at-hand/

28. Spicer J, Tischer B, Peters M. LBA2_PR EGFR mutation testing and oncologist treatment choice in advanced NSCLC: global trends and differences. Ann. Oncol. 26(suppl. 1), i60 (2015). 